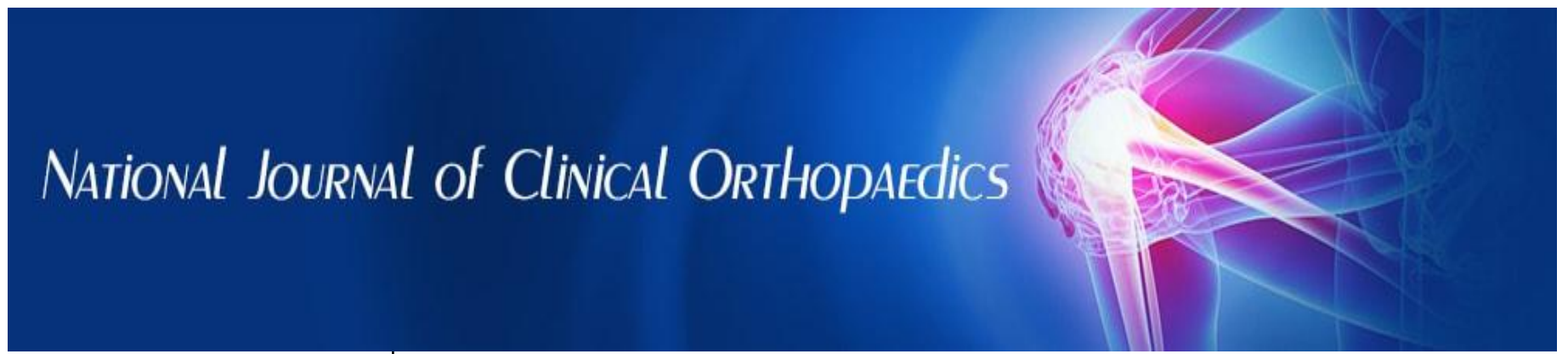

ISSN (P): 2521-3466

ISSN (E): 2521-3474

(C) Clinical Orthopaedics

www.orthoresearchjournal.com

2020; 4(3): 15-18

Received: 12-05-2020

Accepted: 10-07-2020

Dr. Sukumar Nayak

Associate Professor, Department of Physiotherapy, Garden City College, Bengaluru, Karnataka, India

Dr. Manjunatha A

Consultant Orthopedician,

Srinivasa Multispecialty

Hospital, Hoskote, Bengaluru,

Karnataka, India

Dr. Preethish Kumar Thakur PT Consultant Physiotherapist, Srinivasa Multispecialty

Hospital, Hoskote, Bengaluru, Karnataka, India
Corresponding Author: Dr. Manjunatha A Consultant Orthopedician, Srinivasa Multispecialty Hospital, Hoskote, Bengaluru, Karnataka, India

\section{A study to compare the effect of elastic anti pronation taping technique versus space correction taping technique in female subjects with medial tibial stress syndrome}

\author{
Dr. Sukumar Nayak, Dr. Manjunatha A and Dr. Preethish Kumar \\ Thakur PT
}

DOI: https://doi.org/10.33545/orthor.2020.v4.i3a.227

\section{Abstract}

In a foot that is over pronated the movement of the limb takes more energy to propel the weight of the body. This can cause foot and leg fatigue secondary to overuse of muscles. Pes planus feet have been associated with tibialis posterior. One of the primary functions of the tibialis posterior is to contract eccentrically throughout the deceleration phase of foot pronation. If it is dysfunctional it cannot effectively control the force that must be transferred from foot to the lower leg. Leading to its instability control the falling of the arch. Sixty Subjects were selected based on Inclusive and exclusive criteria and an informed consent was taken from the subjects. Pre and post treatment pain sensitivity was taken as perceived by the visual analogue scale. Pre and post treatment score of range of motion of ankle dorsi flexion was taken prior to the treatment by Goniometer.

Keywords: Medial Tibial Stress Syndrome, Goniometer, Pre and Post Treatment Score

\section{Introduction}

In order to diagnosis MTSS, a thorough clinical history and physical examination must be conducted. It will often present as diffuse, palpable pain localized to the postero-medial tibial border. The pain can be located anywhere along this border, however it is commonly found from middle to distal thirds. It is described as a dull ache after exercise. It may last for several hours up to days. MTSS is often diagnosed as shin splints, Shin pain, periostitis, and exercise related lower leg pain. Imaging and diagnosis MTSS with radiograph is not appropriate however, magnetic resonance imaging has increasingly been used for studying MTSS ${ }^{[1]}$.

MTSS is characterized by Exercise induced pain along the postero-medial border of the tibia not attributed to compartment syndrome or stress fracture, a common overuse injury ${ }^{[2]}$.

MTSS seem to be a common link of these varied pathologies with abnormalities in foot position, in particular to a drop in the position of the navicular bone resulting in a fallen arch, a fallen arch is often referred to as pes planus. The foot then is pes planus the ground reaction force point of pressure application also known as the centre of pressure is deviated more medially than those with a neutrally aligned foot ${ }^{[3]}$.

According to the studies low arched individuals have a more medial centre of pressure than those with high arches.

In a foot that is over pronated the movement of the limb takes more energy to propel the weight of the body. This can cause foot and leg fatigue secondary to overuse of muscles. Pes planus feet have been associated with tibialis posterior. One of the primary functions of the tibialis posterior is to contract eccentrically throughout the deceleration phase of foot pronation. If it is dysfunctional it cannot effectively control the force that must be transferred from foot to the lower leg.Leading to its instability control the falling of the arch. The hyper pronation during gait can result in lower leg pain which supports the cause of tibialis posterior weakness which leads to MTSS ${ }^{[4]}$. Currently, there is no acceptable successful treatment plan that consistently alleviates symptoms of MTSS in all population. Many studies describe 
Conservative treatment option for treatment including ice application, aspirin, heel cord stretching and walking cast. There are Studies suggesting ultrasound, iontophoresis, phonophoresis, periosteal packing ${ }^{[5]}$.

As there is a lack of agreement upon the most effective method on MTSS, the need of present study is to know whether tapping along with cryotherapy on application in two different method is going to be effective or not.

Several studies have reported mechanical changes induced by anti-pronation technique, including increased navicular height, decreased calcaneal eversion, and decreased internal tibial rotation in both resting standing posture and during walking and running. ${ }^{6}$ Space correction taping technique is applied to create more space directly above an area of pain, inflammation, swelling, or edema. The increased space that is created decreases pressure by lifting the skin directly over the treatment area. The resulting decreased pressure assists in reducing the amount of irritation on the chemical receptors, thus decreasing pain. An increased level of circulation is also felt to occur in the area, allowing for increased removal of exudates. Stimulation of the mechanoreceptors may also aid in decreasing pain. By increasing sensory stimulation, the gate control theory of pain may be initiated.

\section{Methodology}

Sixty Subjects were selected based on Inclusive and exclusive criteria and an informed consent was taken from the subjects.

A pre and post treatment pain sensitivity was taken as perceived by the visual analogue scale.

Pre and post treatment score of range of motion of ankle dorsi flexion was taken prior to the treatment by Goniometer.

\section{Outcome measures}

Outcome measures of the treatment will be

\section{The Numeric Pain Rating Scale}

It is a segmented numeric version of the visual analog scale (VAS). It is an 11-point numeric scale (NRS 11) with 0 representing no pain and 10 representing extreme pain. The respondent is asked to indicate the numeric value on the segmented scale that best describes their pain intensity. The number that the respondent indicates on the scale to rate their pain intensity is recorded. Scores range from 0-10. Higher scores indicate greater pain intensity.

\section{Goniometer}

The subject will be measured for ankle dorsi-flexion and plantar flexion range of motion using goniometer. The subject should be sitting at the end of couch and the legs will be kept hanging. The stable arm of goniometer will be placed over the midline of the medial aspect of the leg and is hold by the therapist's left hand. The movable arm of goniometer will be placed 90 degree to stable arm and is hold by the therapist's right hand. Therapist's right hand will perform the plantar and dorsi-flexion movement of the ankle and measuring the angle with goniometer to see the passive range of motion and the active range of motion will be measured by subject himself performing the movement.

\section{Description of Treatment}

60 subjects were divided into 2 group- Group 1 and Group 2 .

\section{Group A}

30 subjects received advice and education about the treatment. Following this, elastic anti-pronation taping technique was applied on the affected area continuously for three days followed by cryotherapy for 10 minutes. Subjects were re taped every fourth day for 3 weeks. Subjects were instructed to remove the tape and tape residue the night prior to attending the testing session. At the end of $1^{\text {st }}$ session the subjects were attend a thirty minute physiotherapy session where advice and education was reinforced. Regular training was commenced.

\section{Group B}

30 subjects received advice and education about the treatment. Following this, space correction taping technique was applied on the affected area continuously for three days followed by cryotherapy for 10 minutes. Subjects were re taped every fourth day for 3 weeks. Subjects were be instructed to remove the tape and tape residue the night prior to attending the testing session. At the end of $1^{\text {st }}$ session the subjects attended a thirty minute physiotherapy session where advice and education was reinforced. Regular training was commenced.

\section{Application of elastic anti-pronation tape}

$\mathrm{K}$-tape is applied by cutting out a small wedge from one end to allow it to conform to the proximal phalanx of the first toe. With foot and ankle in full plantar flexion, inversion, adduction and flexion of the first toe, the tape is applied from the plantar surface of the first toe, along the medial plantar aspect of the foot, onto the medial aspect of the calcaneus, around the calcaneus to the cross the lateral aspect obliquely, continuing under the plantar aspect of the foot to immerged under the navicular and finally lifting the navicular to anchor the tape on the dorsum of foot.

Then applied with and ankle in full dorsiflexion, inversion, and adduction the tape is applied from the plantar surface of the foot between the first and second metatarsal, courses over the dorsum of the foot from medial to lateral, under the plantar aspect of the foot on diagonal to the navicular. Lifting the navicular coursing across the anterior anteriotalocural joint to anchor on the lateral aspect of the mid-shin region.

\section{Application of space correction tape}

Space correction method tissue away: this method pulls skin away from the medial border of the tibia. Begins by placing the kinesio y strip just inferior to the area of pain. Hold the base of the kinesio tape to avoid unwanted tension on the skin. Apply light to moderate tension and pull the skin away from the medial tibial border, laying down the strip as it continues to move more inferiorly. When tension is applied to the point here the kinesio tape $y$, slide the hand holding the base to the initiation of the $y$ cut. Have the subject dorsiflex their ankle, and apply the tails of the $\mathrm{y}$ with to tension in splayed out pattern. 


\section{Results}

Table 1: Comparison of two techniques (Anti pronation taping and Space correction taping) with respect to mean age and BMI scores by $t$ test

\begin{tabular}{|c|c|c|c|c|c|c|}
\hline Variable & Techniques & Mean & Sd & Se & T-Value & P-Value \\
\hline Age in yrs & Anti-pronation taping & 26.67 & 1.42 & 0.26 & 1.9248 & 0.0592 \\
\hline & Space correction taping & 26.07 & 0.94 & 0.17 & & \\
\hline Height in cms & Anti-pronation taping & 134.29 & 2.84 & 0.52 & -1.1378 & 0.2599 \\
\hline & Space correction taping & 135.10 & 2.72 & 0.50 & & \\
\hline Weight in kgs & Anti-pronation taping & 53.08 & 3.47 & 0.63 & 0.9994 & 0.3218 \\
\hline & Space correction taping & 52.14 & 3.79 & 0.69 & & \\
\hline BMI & Anti-pronation taping & 29.45 & 2.01 & 0.37 & 1.6176 & 0.1112 \\
\hline & Space correction taping & 28.58 & 2.14 & 0.39 & & \\
\hline
\end{tabular}

Table 2: Comparison of two techniques (Anti pronation taping and Space correction taping) with respect to pretest and posttest Dorsi Flexion scores by Mann-Whitney U test

\begin{tabular}{|c|c|c|c|c|c|c|c|}
\hline Time points & Techniques & Mean & SD & Sum of ranks & U-value & Z-value & P-value \\
\hline Pretest & Anti-pronation taping & 15.10 & 1.84 & 1001.50 & & & \\
\hline & Space correction taping & 14.60 & 1.69 & 828.50 & 363.50 & -1.2789 & 0.2010 \\
\hline Posttest & Anti-pronation taping & 18.37 & 1.00 & 1310.00 & & & \\
\hline & Space correction taping & 15.40 & 1.54 & 520.00 & 55.00 & -5.8398 & $0.0001^{*}$ \\
\hline Difference & Anti-pronation taping & 3.27 & 1.41 & 1342.50 & & & \\
\hline & Space correction taping & 0.80 & 0.61 & 487.50 & 22.50 & -6.3203 & $0.0001^{*}$ \\
\hline$* p<0.05$
\end{tabular}

Table 3: Comparison of two techniques (Anti pronation taping and Space correction taping) with respect to pretest and posttest Planter Flexion scores by Mann-Whitney U test

\begin{tabular}{|c|c|c|c|c|c|c|c|}
\hline Time points & Techniques & Mean & SD & Sum of ranks & U-value & Z-value & P-value \\
\hline Pretest & Anti pronation taping & 50.13 & 4.38 & 926.50 & & & \\
\hline & Space correction taping & 50.37 & 3.76 & 903.50 & 438.50 & -0.1700 & 0.8650 \\
\hline Posttest & Anti pronation taping & 54.40 & 3.86 & 1149.00 & & & \\
\hline & Space correction taping & 51.30 & 3.56 & 681.00 & 216.00 & -3.4596 & $0.0005^{*}$ \\
\hline Difference & Anti pronation taping & 4.27 & 1.82 & 1340.50 & & & \\
\hline$* p<0.05$ & Space correction taping & 0.93 & 0.64 & 489.50 & 24.50 & -6.2908 & $0.0001^{*}$ \\
\hline
\end{tabular}

Table 4: Comparison of two techniques (Anti pronation taping and Space correction taping) with respect to pretest and posttest pain scores by Mann-Whitney U test

\begin{tabular}{|c|c|c|c|c|c|c|c|}
\hline Time points & Techniques & Mean & SD & Sum of ranks & U-value & Z-value & P-value \\
\hline Pretest & Anti-pronation taping & 6.13 & 0.86 & 921.50 & & & \\
\hline & Space correction taping & 6.13 & 0.82 & 908.50 & 443.50 & -0.0961 & 0.9234 \\
\hline Posttest & Anti-pronation taping & 2.87 & 1.11 & 520.00 & & & \\
\hline & Space correction taping & 5.27 & 0.83 & 1310.00 & 55.00 & -5.8398 & $0.0001 *$ \\
\hline Difference & Anti-pronation taping & 3.27 & 1.31 & 1293.00 & & & \\
\hline & Space correction taping & 0.87 & 0.78 & 537.00 & 72.00 & -5.5885 & $0.0001 *$ \\
\hline$p<0.05$
\end{tabular}

\section{Discussion}

Golam Reza D Raissi, et al. conducted a study to determine the relationship between lower extremity alignment and MTSS amongst non-professional athletes. They concluded that there is a significant relationship between navicular drop and MTSS ${ }^{[7]}$.

Debbie I. Craig, et al. conducted a study on medial tibial stress syndrome: evidence based Prevention. Prevention methods studied were shock absorbent insoles, foam heel pads, Achilles tendon stretching, footwear, and graduated running programs. No statistically significant results were noted for any of the prevention methods ${ }^{[5]}$.

S G Burne, et al conducted a study on Risk factors associated with exertional medial tibial pain: a 12 month prospective clinical study. They concluded that Greater internal and external hip range of motion and lower lean calf girth was associated with exertional medial tibial pain in male military cadets. Women had high rates of injury, although no intrinsic factor was identified. Reasons for this sex difference need to be identified
[6].

Ben Yates, et al. conducted a study on The Incidence and Risk Factors in the Development of Medial Tibial Stress Syndrome among Naval Recruits. To identify the incidence of medial tibial stress syndrome (MTSS) in a group of naval recruits undergoing a 10 -week basic training period and to determine potential risk factors. They concluded that identifying a pronated foot type prior to training may help reduce the incidence of medial tibial stress syndrome by early intervention to control abnormal pronation ${ }^{[8]}$

James U Youdus, conducted a study Reliability of goniometric measurements and visual estimates of ankle joint active range of motion obtained in a clinical setting. They concluded that range of motion measurements of ankle dorsi flexion and plantar flexion made by the same physical therapist using universal goniometer have a good degree of reliability ${ }^{[9]}$.

Wayne Smith, et al conducted a study to compare the effects of ice massage, ultrasound, iontophoresis, and phonophoresis in 
young adults with a shinsplint syndrome. Fifty patients from a military recruit population with shinsplint syndrome were randomly assigned into 5 groups of 10 . The results indicated that none of these treatment modalities was superior to another. However, all were clearly superior to a controlled treatment $\operatorname{program}^{[10]}$.

\section{References}

1. Kase K. Clinical therapeutic applications of the kinesio taping method. $2^{\text {nd }}$ ed. USA: Kineso Taping Assoc. 2003; 29:149.

2. Menz HB, Dufour AB, Riskowski JL, Hillstrom HJ, Hannan MT. Association of planus foot posture and pronated foot function with foot pain: the Framingham foot study. Arthritis care \& research. 2013; 65(12):1991-9.

3. Moen MH, Holtslag L, Bakker E, Barten C, Weir A, Tol JL, et al. The treatment of medial tibial stress syndrome in athletes; a randomized clinical trial. Sports Medicine, Arthroscopy, Rehabilitation, Therapy \& Technology. 2012; 4(1): 12 .

4. Raissi GR, Cherati AD, Mansoori KD, Razi MD. The relationship between lower extremity alignment and Medial Tibial Stress Syndrome among non-professional athletes. BMC Sports Science, Medicine and Rehabilitation. 2009; 1(1): 11 .

5. Craig DI. Medial tibial stress syndrome: evidence-based prevention. Journal of athletic training. 2008; 43(3):316-8.

6. Burne SG, Khan KM, Boudville PB, Mallet RJ, Newman PM, Steinman LJ et al. Risk factors associated with exertional medial tibial pain: a 12 month prospective clinical study. British journal of sports medicine. 2004; 38(4):441-5.

7. Winters M, Eskes M, Weir A, Moen MH, Backx FJ, Bakker EW et al. Treatment of medial tibial stress syndrome: a systematic review. Sports Medicine. 2013; 43(12):1315-33.

8. Yates $\mathrm{B}$, White $\mathrm{S}$. The incidence and risk factors in the development of medial tibial stress syndrome among naval recruits. The American journal of sports medicine. 2004; 32(3):772-80.

9. Youdas JW, Bogard CL, Suman VJ. Reliability of goniometric measurements and visual estimates of ankle joint active range of motion obtained in a clinical setting. Archives of physical medicine and rehabilitation. 1993; 74(10):1113-8.

10. Smith W, Winn F, Parette R. Comparative study using four modalities in shinsplint treatments. Journal of Orthopaedic \& Sports Physical Therapy. 1986; 8(2):77-80. 